\title{
Exogenous hydrogen sulfide reduces atrial remodeling and atrial fibrillation induced by diabetes mellitus via activation of the PI3K/Akt/eNOS pathway
}

\author{
XIAOFEI XUE ${ }^{1 *}$, XINYU LING $^{1 *}$, WANG XI ${ }^{1}$, PEI WANG ${ }^{1}$, JIANJUN SUN $^{2}$, QIAN YANG ${ }^{1}$ and JIAN XIAO ${ }^{1}$ \\ ${ }^{1}$ Center for Comprehensive Treatment of Atrial Fibrillation, Department of Cardiothoracic Surgery, \\ Changzheng Hospital, Second Military Medical University, Shanghai $200003 ;{ }^{2}$ Department of Rehabilitation, \\ Elderly Rehabilitation Hospital, Suzhou Red Cross Society, Suzhou, Jiangsu 215009, P.R. China
}

Received December 18,2019; Accepted May 14, 2020

DOI: $10.3892 / \mathrm{mmr} .2020 .11291$

\begin{abstract}
Diabetes mellitus (DM) facilitates atrial fibrosis and increases the risk of atrial fibrillation (AF). The underlying mechanism of DM in causing AF remains mostly unknown and potential therapeutic targets for DM-induced AF are rarely reported. Hydrogen sulfide $\left(\mathrm{H}_{2} \mathrm{~S}\right)$ has drawn considerable attention in recent years for its potential as a cardiovascular protector. Thus, the aim of the present study was to investigate the effect of $\mathrm{H}_{2} \mathrm{~S}$ on DM-induced $\mathrm{AF}$ and the mechanism of action. Sprague-Dawley rats were divided into four groups, including the control group, the DM group, the $\mathrm{H}_{2} \mathrm{~S}$ group and the $\mathrm{DM}+\mathrm{H}_{2} \mathrm{~S}$ group. The DM group and the $\mathrm{DM}+\mathrm{H}_{2} \mathrm{~S}$ group were administered streptozotocin to induce DM, whereas the other two groups were given citrate buffer as a control. The $\mathrm{H}_{2} \mathrm{~S}$ group and the $\mathrm{DM}+\mathrm{H}_{2} \mathrm{~S}$ group were administered with an intraperitoneal injection of sodium hydrosulfide (precursor of $\mathrm{H}_{2} \mathrm{~S}$ ). AF inducibility, AF duration, atrial fibrosis and vital protein expression of oxidative stress were compared among the four groups. The DM group showed significantly higher AF incidence rates and duration $(\mathrm{P}<0.05)$. Histology results demonstrated severe atrial fibrosis in the DM group, and the PI3K/Akt/endothelial nitric oxide synthase (eNOS) pathway was significantly downregulated $(\mathrm{P}<0.05)$. However, when $\mathrm{H}_{2} \mathrm{~S}$ was administered, the rats showed lower AF incidence and duration compared with the DM group. Additionally, $\mathrm{H}_{2} \mathrm{~S}$ was able to mitigate the atrial fibrosis induced by DM,
\end{abstract}

Correspondence to: Dr Jian Xiao or Dr Qian Yang, Center for Comprehensive Treatment of Atrial Fibrillation, Department of Cardiothoracic Surgery, Changzheng Hospital, Second Military Medical University, 415 Fengyang Road, Huangpu, Shanghai 200003, P.R. China

E-mail: 18916066266@189.cn

E-mail: yangqiandoc@163.com

*Contributed equally

Key words: hydrogen sulfide, atrial fibrosis, atrial fibrillation, diabetes mellitus, PI3K/Akt/endothelial nitric oxide synthase pathway as well as the proliferation and migration of cardiac fibroblasts, as demonstrated by an MTT assay and real-time cell analyzer migration experiment. Western blotting showed that the expression levels of the PI3K/Akt/eNOS pathway in the $\mathrm{DM}+\mathrm{H}_{2} \mathrm{~S}$ group were significantly upregulated compared with those of the DM group $(\mathrm{P}<0.05)$. In summary, DM status can lead to the structural remodeling of atrial fibrosis, facilitating AF incidence and persistence. Administration of $\mathrm{H}_{2} \mathrm{~S}$ does not affect the glucose level, but can significantly mitigate atrial fibrosis and reduce the incidence of AF induced by DM, probably via activation of the PI3K/Akt/eNOS pathway.

\section{Introduction}

As the most common sustained arrhythmia diagnosed in the clinic, atrial fibrillation (AF) significantly increases the mortality and morbidity of patients by impairing cardiac function and causing stroke (1). Epidemiological studies have demonstrated that diabetes mellitus (DM) is one of the major risk factors leading to the pathogenesis of AF, which increases the risk by $\sim 40 \%$ (2). It was previously reported that autonomic, electrical, electromechanical and structural remodeling, including oxidative stress, connexin remodeling and glycemic fluctuations, triggered by DM, contribute to AF pathophysiology (3). However, the underlying mechanism of the mutual relationship between AF and DM remains mostly unknown, which limits the prevention and treatment of DM-induced AF. Streptozotocin (STZ) is a natural chemical, which can be taken up by pancreatic B cells via the glucose transporter and exert toxic effects. Due to its distinctive effect, STZ is widely applied as a tool to induce experimental DM animal models (4). Hayami et al (5) investigated the impact of a DPP-4 inhibitor on AF in a rat model induced by STZ, and found that the administration of STZ greatly increased atrial fibrosis, but sulfonylurea and DPP-4 inhibitors inhibited inflammation and fibrosis of the atria.

Previous research has demonstrated that inflammation and oxidative stress play a critical role in the relationship between DM and AF (6). Therefore, agents mitigating inflammation and oxidative stress might be useful in preventing DM-induced AF. Hydrogen sulfide $\left(\mathrm{H}_{2} \mathrm{~S}\right)$ has drawn considerable attention, not only due to its inherent activity in human physiology, but 
also because of its potential as a cardiovascular protector (7). Most studies of $\mathrm{H}_{2} \mathrm{~S}$ and cardiovascular disorders have focused on myocardial ischemia/reperfusion injury rather than arrhythmia, especially AF (7,8). In addition, there is evidence that downregulated PI3K-Akt-eNOS expression is related to an increased AF inducibility in diabetic rats (9). In addition, it has been noted that $\mathrm{H}_{2} \mathrm{~S}$ is associated with inflammation and oxidative-stress responses $(10,11)$. In the present study, it was hypothesized that $\mathrm{H}_{2} \mathrm{~S}$ could reverse $\mathrm{AF}$ and reduce the incidence of AF in diabetic rats via the PI3K-Akt-eNOS signaling pathway, so the effect of $\mathrm{H}_{2} \mathrm{~S}$ on DM-induced AF and its underlying mechanisms were explored.

\section{Materials and methods}

Animal model. A total of 80 adult Sprague-Dawley (SD) rats were used in this study, which was approved by the Experimental Animal Administration Committee of the Second Military Medical University (Shanghai, China). Animal experiments were performed following the Guide for the Care and Use of Laboratory Animals (National Institutes of Health publication 85-23, revised 1996). The 12-week-old male rats (weight 200-220 g) were randomly divided into the control group, the DM group, the $\mathrm{H}_{2} \mathrm{~S}$ group, and the $\mathrm{DM}+\mathrm{H}_{2} \mathrm{~S}$ group (each group included 20 rats). All rats were fed a routine diet and maintained in a standard room environment $\left(18-23^{\circ} \mathrm{C}\right.$, humidity 40-60\%, 12-h light/dark cycle, and free access to food and water). Animal health and behavior were monitored and evaluated every week, including general condition, appetite, sleep status and survival.

Rats of the DM group and the $\mathrm{DM}+\mathrm{H}_{2} \mathrm{~S}$ group were administered STZ (60 mg/kg; Wako Chemicals USA, Inc.) dissolved in citrate buffer (Sigma-Aldrich; Merck KGaA) intraperitoneally (i.p.) at the age of 12 weeks. After one week, the rats were tested for fasting blood glucose (FBG) level. The fasting time was $16 \mathrm{~h}$. If the FBG level was $>200 \mathrm{mg} / \mathrm{dl}$, then the rats were selected for the following experiments (5). At the same time, the control group and the $\mathrm{H}_{2} \mathrm{~S}$ group were administered citrate buffer only. After the injection, the $\mathrm{H}_{2} \mathrm{~S}$ group and the $\mathrm{DM}+\mathrm{H}_{2} \mathrm{~S}$ group were administered $15 \mu \mathrm{mol} / \mathrm{kg}$ (i.p.) sodium hydrosulfide (NaHS, exogenous $\mathrm{H}_{2} \mathrm{~S}$ donor; Sigma-Aldrich; Merck KGaA) every week for 8 consecutive weeks, whereas the control group and the DM group were administered normal saline only. At the age of 20 weeks, all rats were subjected to the following experiments.

Intraperitoneal glucose tolerance test (IPGTT) and intraperitoneal insulin tolerance test (IPITT). As previously reported (12), IPITT and IPGTT were conducted at the age of 20 weeks. In terms of IPGTT, the rats were fasted overnight for $15 \mathrm{~h}$, and were given $1.5 \mathrm{gr} / \mathrm{kg}$ glucose through an i.p. injection. Blood was collected from the tail vein for glucose measurements at $0,10,20,30,60,90$ and $120 \mathrm{~min}$ after glucose injection. As for IPITT, the rats received $2.5 \mathrm{U} / \mathrm{kg}$ insulin via i.p. injection, and then blood was drawn from the tail vein for glucose measurements at $0,20,30,40,60,90$ and $120 \mathrm{~min}$ after insulin administration.

Intracardiac programmed electrical stimulus. As previously described, all groups were examined with electrophysiological characteristics via intracardiac programmed electrical stimulus (13). Each animal was anesthetized with $2 \%$ isoflurane (Beijing Hongxin Technology) inhalation and fixed on a heated pad at the prone position to maintain $37^{\circ} \mathrm{C}$ body temperature. Surface electrocardiogram (ECG; B\&W TEKSystems, Inc.) and intracardiac electrograms were recorded simultaneously. $\mathrm{P}$ wave duration and an indicator of intra-atrial conduction time were measured. An electrode catheter (1.1 F; Millar) was placed into the right atrium through the right jugular vein. A burst of electrical stimuli for $30 \mathrm{sec}$ was used to test the inducibility of atrial arrhythmias. The burst test was conducted 3 times, and $>$ two incidents of AF observed ( $P$ wave disappeared and lasted $>2 \mathrm{sec}$ ) was judged as the onset of AF. LabChart Software 7.0 (ADInstruments) was used to record and analyze electrocardiogram data.

Western blot analysis. As previously described (14), expression levels of key proteins of the PI3K/Akt/endothelial nitric oxide synthase (eNOS) pathway in the atrial myocardium were examined with western blotting. The membranes were incubated with the following primary antibodies overnight at $4^{\circ} \mathrm{C}$, anti-PI3K (1:2,000, cat. no. ab151549; Abcam), anti-Akt (1:2,000, cat. no. ab8805; Abcam), anti-phosphorylated (p)-Akt (1:500, cat. no. 9611; Cell Signaling Technology, Inc.), anti-p-eNOS (1:500, cat. no. ab184154; Abcam), anti-eNOS (1:500, cat. no. ab76198, Abcam) and anti- $\beta$-actin (1:5,000, cat. no. ab179467; Abcam). Following the primary incubation, membranes were incubated with rabbit anti-mouse (1:5,000, cat. no. ab150113; Abcam) or goat anti-rabbit HRP-conjugated secondary antibody (1:5,000, cat. no. ab150077; Abcam). Gel Imaging system (Bio-Rad Laboratories, Inc.) and ImageJ software (Version 1.8, National Institutes of Health) were used to image and analyze the western blotting bands.

Reverse transcription-quantitative $(R T-q) P C R$. Total RNA from atrial myocardium samples was extracted using TRIzol (Takara Biotechnology Co., Ltd.). Total RNA (1,000 ng) was reverse transcribed into cDNA using the Prime-Script ${ }^{\mathrm{TM}}$ RT Reagent kit (Takara Biotechnology Co., Ltd.). Before performing the RT reaction, RNA $(10 \mu \mathrm{l})$ was heated to $65^{\circ} \mathrm{C}$ for 5-10 $\mathrm{min}$, and then quenched on ice. Then, the inversion system was prepared according to the instructions of the cDNA kit, the sample was placed at $25^{\circ} \mathrm{C}$ for $10 \mathrm{~min}$ and then incubated at $42^{\circ} \mathrm{C}$ for $1 \mathrm{~h}$. Following which, the cDNA was denatured at $95^{\circ} \mathrm{C}$ and placed on ice.

RT-qPCR was performed using SYBR-Green (Takara Biotechnology Co., Ltd.) and normalized to $\beta$-actin expression. The fibroblast-to-myofibroblast transition marker $\alpha$-smooth muscle actin, and atrial fibrosis markers, transforming growth factor (TGF)- $\beta$, collagen I, collagen III, were examined. A total of 30 cycles of PCR were performed: Denaturation at $95^{\circ} \mathrm{C}$ for $30 \mathrm{sec}$, annealing at $60^{\circ} \mathrm{C}$ for $45 \mathrm{sec}$, and extension at $72^{\circ} \mathrm{C}$ for $60 \mathrm{sec}$.

The primer sequences are listed as follows: Akt, 5'-AGC ATGGAGTGTGTGGACAG-3' (forward) and 5'-GATGAT CCATGCGGGGCTT-3' (reverse); eNOS, 5'-CGACTATCC TGTATGGCTCTGAG-3' (forward) and 5'-GATCCCCAT TGCCAAATGTGC-3' (reverse); PI3K, 5'-GCTCTTTCCCCA GCTGAACT-3' (forward) and 5'-ACACAGTGTCGCTGGTTT GA-3' (reverse); $\beta$-actin: 5'-CATCCTGCGTCTGGACCGG-3' 
(forward) and 5'-TAATGTCACGCACGATTTCC-3' (reverse); TGF- $\beta, 5$ '-CCCAGCATCTGCAAAGCTC-3' (forward) and 5'-GTCAATGTACAGCTGCCGCA-3' (reverse); collagen I, 5'-CATGTTCAGCTTTGTGGACCT-3' (forward) and 5'-GCA GCTGACTTCAGGGATGT-3' (reverse). The average expression of $\beta$-actin was used as an internal reference gene to normalize input cDNA. Relative gene expression was calculated via the threshold cycle value (CT) and the formula $2^{-\Delta \Delta C q}(15)$. All of the reactions were repeated at least twice, with at least 3 replicates for every sample.

Masson's trichrome staining. According to a previous report (16), Masson's trichrome staining was conducted to evaluate the extent of atrial fibrosis. After the rats were sacrificed, atrium samples were rapidly excised, rinsed with PBS, fixed in $4 \%$ paraformaldehyde at $4^{\circ} \mathrm{C}$ overnight, and embedded in paraffin. Then, $5-\mu \mathrm{m}$-thick sections at of the atrium were cut. Masson's trichrome kit (cat. no. HT15; Sigma-Aldrich; Merck KGaA) was used according to the manufacturer's instruction. Image J software (Version 1.8; National Institutes of Health) was used to evaluate the area of fibrosis. Each section was examined in 5 randomly-selected high-power fields (x400) and middle-power fields (x200) under a light microscope. Collagen volume fraction $(\mathrm{CVF})=$ Collagen area (blue)/Total area $\times 100 \%$.

Cell culture. Cardiac fibroblasts (CFs) were harvested from the different groups, according to a previous report (17). Following attachment to uncoated culture plates, all unattached or weakly attached cells were removed. Then, the attached fibroblasts were washed and grown in DMEM (Gibco; Thermo Fisher Scientific, Inc.) supplemented with 10\% FBS (Gibco; Thermo Fisher Scientific, Inc.) at $37^{\circ} \mathrm{C}$. CFs were plated on a 96 -well plate at a density of $1 \times 10^{4} /$ well and cultured for $24 \mathrm{~h}$.

Cell proliferation assay. As previously described (18), cell proliferation was examined using an MTT assay. Data values are expressed as fold-change compared with the control group.

Cell migration. As previously described (18), cell migration was examined with the real-time cell analyzer (RTCA) migration experiment. The CFs were seeded in RTCA Cim-16 plates in a serum-free DMEM medium with a cell density of at least 20,000/well. A chemoattractant of full growth medium (DMEM with 5-10\% bovine serum albumin; cat. no. A1933; Sigma-Aldrich; Merck KGaA) was applied in the lower chamber. The RTCA device (xCELLigence RTCA; Roche Diagnostics) was used for measurements in a time-resolved manner (18). The values are expressed as fold-change compared with the control group.

Statistical analysis. GraphPad Prism 7.0 (GraphPad Software, Inc.) was used for statistical analysis. The AF incidence of four groups was compared with the Chi-square test. For comparison of normally distributed variables, one-way ANOVA and Tukey's post hoc test was performed. For comparison of AF duration, Kruskal-Wallis test and Dunn's multiple comparisons post hoc test were conducted. Data are presented as the mean $\pm \mathrm{SD}$. $\mathrm{P}<0.05$ was considered to indicate a statistically significant difference.

\section{Results}

DM model results. As demonstrated in Fig. 1, the FBG value was evaluated in the four groups at different points, including before the STZ injection, 1, 4 and 8 weeks after STZ injection. Before the injection, there was no difference in FBG among the four groups $(\mathrm{P}=0.5204)$, and the $\mathrm{FBG}$ varied between 90 and $110 \mathrm{mg} / \mathrm{dl}$ (Fig. 1A). However, 1 week after the STZ injection, the DM group and the $\mathrm{DM}+\mathrm{H}_{2} \mathrm{~S}$ group demonstrated a significant increase in the FBG level to $410.8 \pm 92.0$ and $454.1 \pm 93.4 \mathrm{mg} / \mathrm{dl}$, respectively, which were significantly higher than the control group and the $\mathrm{H}_{2} \mathrm{~S}$ group $(\mathrm{P}<0.0001)$. At the same time, there was no significant difference between the $\mathrm{DM}$ and $\mathrm{DM}+\mathrm{H}_{2} \mathrm{~S}$ group, and between the control group and the $\mathrm{H}_{2} \mathrm{~S}$ group (both $\mathrm{P}>0.05$; Fig. 1B). The FBG level was examined 4 and 8 weeks after STZ injection, when NaHS had been administered. The results presented similar trends with the FBG level 1 week after STZ injection. The FBG of the DM group and the $\mathrm{DM}+\mathrm{H}_{2} \mathrm{~S}$ group was significantly higher than the control group and the $\mathrm{H}_{2} \mathrm{~S}$ group $(\mathrm{P}<0.0001)$. At the same time, there was no difference between the DM group and the $\mathrm{DM}+\mathrm{H}_{2} \mathrm{~S}$ group, the control group and the $\mathrm{H}_{2} \mathrm{~S}$ group (both $\mathrm{P}>0.05$ ) (Fig. 1C and D).

IPITT and IPGTT (Fig. 1E and F) was performed to test insulin tolerance and glucose tolerance. The results were consistent with the FBG test, showing that the DM group and the $\mathrm{DM}+\mathrm{H}_{2} \mathrm{~S}$ group shared similar insulin and glucose tolerance, which was notably higher than the control group and the $\mathrm{H}_{2} \mathrm{~S}$ group. However, there was no difference between the control group and the $\mathrm{H}_{2} \mathrm{~S}$ group, and no difference $\mathrm{DM}+\mathrm{H}_{2} \mathrm{~S}$ group and the DM group. No significant side effects of NaHS were observed in the $\mathrm{H}_{2} \mathrm{~S}$ group and the $\mathrm{DM}+\mathrm{H}_{2} \mathrm{~S}$ group.

AF inducibility and persistence. As shown in Fig. 2, the intracardiac programmed electrical stimulus was applied to examine the AF inducibility of the four groups. Fig. 2A shows representative ECG figures of burst pacing and the subsequent heart rhythm. Compared with the other three groups, the DM group exhibited a much higher susceptibility of induced AF, with a significantly higher AF incidence $(\mathrm{P}=0.017)$. There was no statistical difference between the control group, the $\mathrm{H}_{2} \mathrm{~S}$ group and the $\mathrm{DM}+\mathrm{H}_{2} \mathrm{~S}$ group in $\mathrm{AF}$ incidence (Fig. 2B). The $\mathrm{AF}$ duration of the DM group was significantly higher than the control group and the $\mathrm{H}_{2} \mathrm{~S}$ group $(\mathrm{P}=0.005)$. Although the AF duration of the $\mathrm{DM}+\mathrm{H}_{2} \mathrm{~S}$ group was lower than the DM group, there was no statistical significance $(\mathrm{P}=0.085$; Fig. $2 \mathrm{C})$.

Atrial fibrosis. Atrial remodeling of the four groups was also evaluated using Masson's trichrome staining (Fig. 3). More blue-stained collagen was observed in the DM group, indicating a more severe atrial fibrosis than the other groups, whereas the $\mathrm{DM}+\mathrm{H}_{2} \mathrm{~S}$ group suffered less fibrosis than the DM group (Fig. 3A). Fibrosis was quantified the with ImageJ software. The CVF value was calculated for the four groups, which indicated that the CVF of the DM group was significantly higher than the other groups $(\mathrm{P}<0.0001)$. At the same time, there was no significant difference among the control group, the $\mathrm{H}_{2} \mathrm{~S}$ group and the $\mathrm{DM}+\mathrm{H}_{2} \mathrm{~S}$ group (all $\mathrm{P}>0.05$; Fig. 3B). The key markers of fibrosis, including TGF- $\beta$ and Collagen I, were also determined (Fig. $3 \mathrm{C}$ and $\mathrm{D}$ ), which demonstrated that TGF- $\beta$ 
A

Before STZ

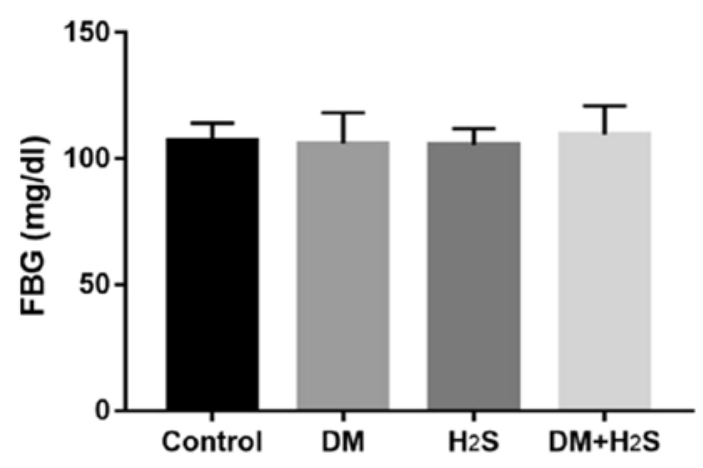

C 4 weeks after STZ

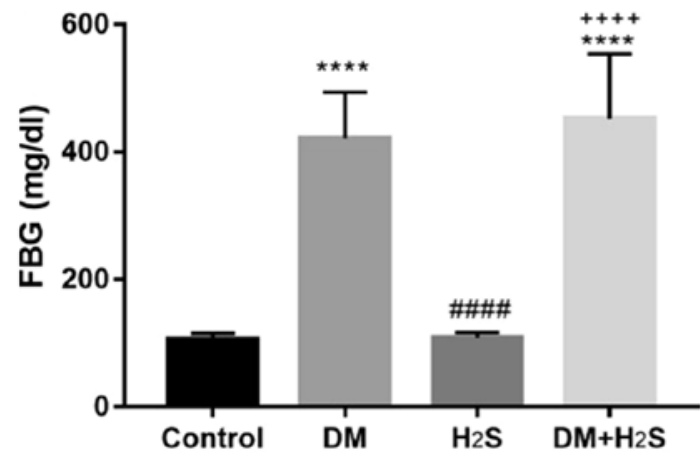

E

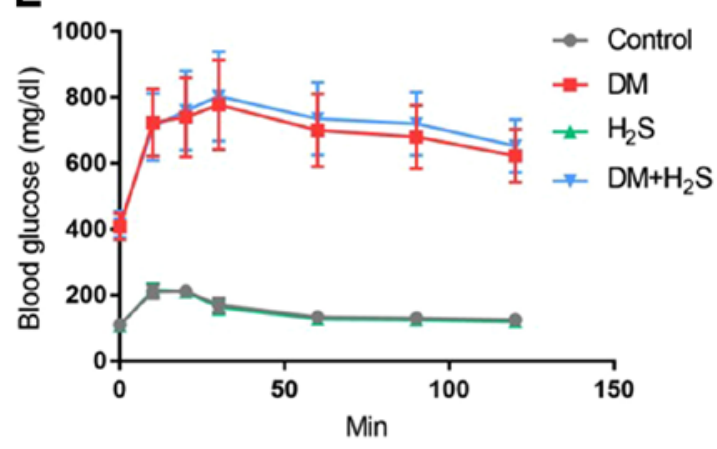

B 1 week after STZ

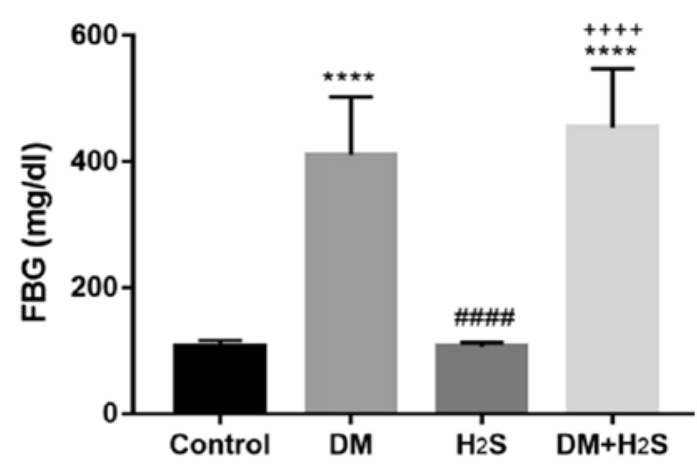

D 8 weeks after STZ

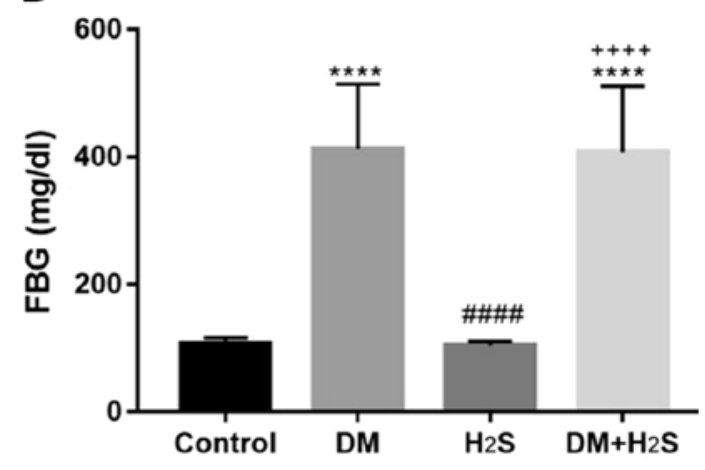

IPITT

$\mathbf{F}$

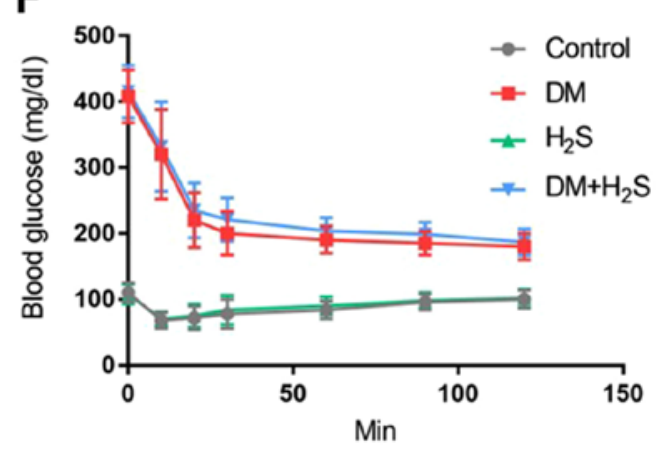

Figure 1. FBG level in the four groups at different time points. (A) FBG level before STZ injection. (B) FBG level at 1 week after STZ injection. (C) FBG level at 4 weeks after STZ injection. (D) FBG level at 8 weeks after STZ injection. (E) IPGTT results of the four groups. (F) IPITT results of the four groups. $\mathrm{n}=20$

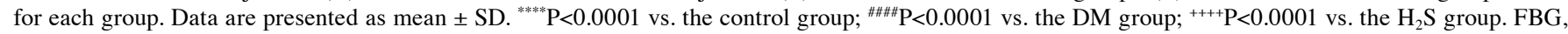
fast blood glucose; STZ, streptozotocin; DM, diabetes mellitus; $\mathrm{H}_{2} \mathrm{~S}$, hydrogen sulfide; IPGTT, Intraperitoneal glucose tolerance test; IPITT, intraperitoneal insulin tolerance test.

and Collagen I of the DM group was significantly higher than the other groups $(\mathrm{P}<0.0001)$. Fibrosis of the different groups was also examined at the cellular level by comparing the proliferation and migration of CFs. CF proliferation and migration of the DM group was significantly increased. In contrast, proliferation and migration of the $\mathrm{DM}+\mathrm{H}_{2} \mathrm{~S}$ group were lower than the DM group $(\mathrm{P}<0.0001$; Fig. $3 \mathrm{E}$ and $\mathrm{F})$.

PI3K/Akt/eNOS signaling pathway. As shown in Fig. 4, the classic PI3K/Akt/eNOS signaling pathway was evaluated in the four groups with RT-qPCR (Fig. 4A-C) and western blot analysis (Fig. 4D-G). The results demonstrated that this pathway was significantly downregulated in the DM group compared with the control group, which was reversed in the $\mathrm{DM}+\mathrm{H}_{2} \mathrm{~S}$ group. The expression levels of PI3K/Akt/eNOS were significantly higher in the $\mathrm{DM}+\mathrm{H}_{2} \mathrm{~S}$ group $(\mathrm{P}<0.0001)$. Compared with the DM group, p-Akt/Akt and p-eNOS/eNOS ratios were significantly increased in the $\mathrm{DM}+\mathrm{H}_{2} \mathrm{~S}$ group (Fig. 4F and G).

\section{Discussion}

$\mathrm{H}_{2} \mathrm{~S}$, an endogenous active molecule, has been demonstrated to be effective in organ protection for ischemia/reperfusion injury (8). However, data concerning its effect on AF incidence, especially on DM-induced AF, is minimal. In the 
A

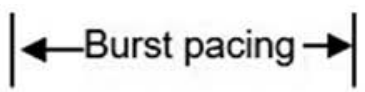

Control

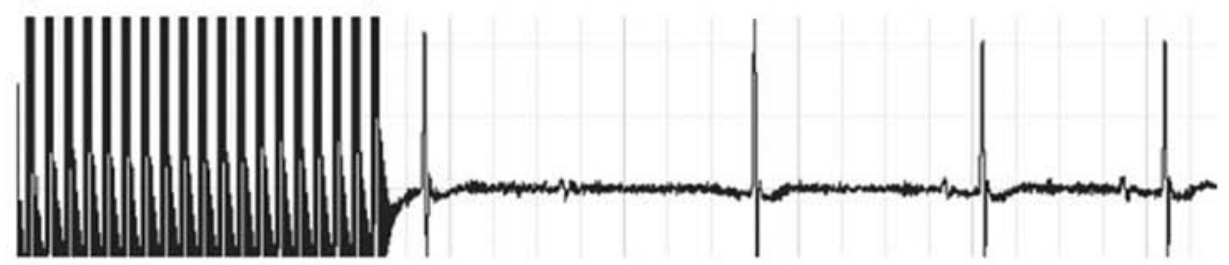

DM

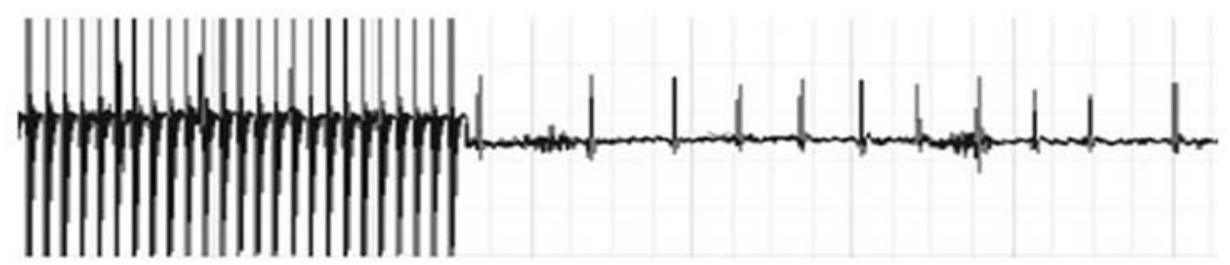

$\mathrm{H}_{2} \mathrm{~S}$
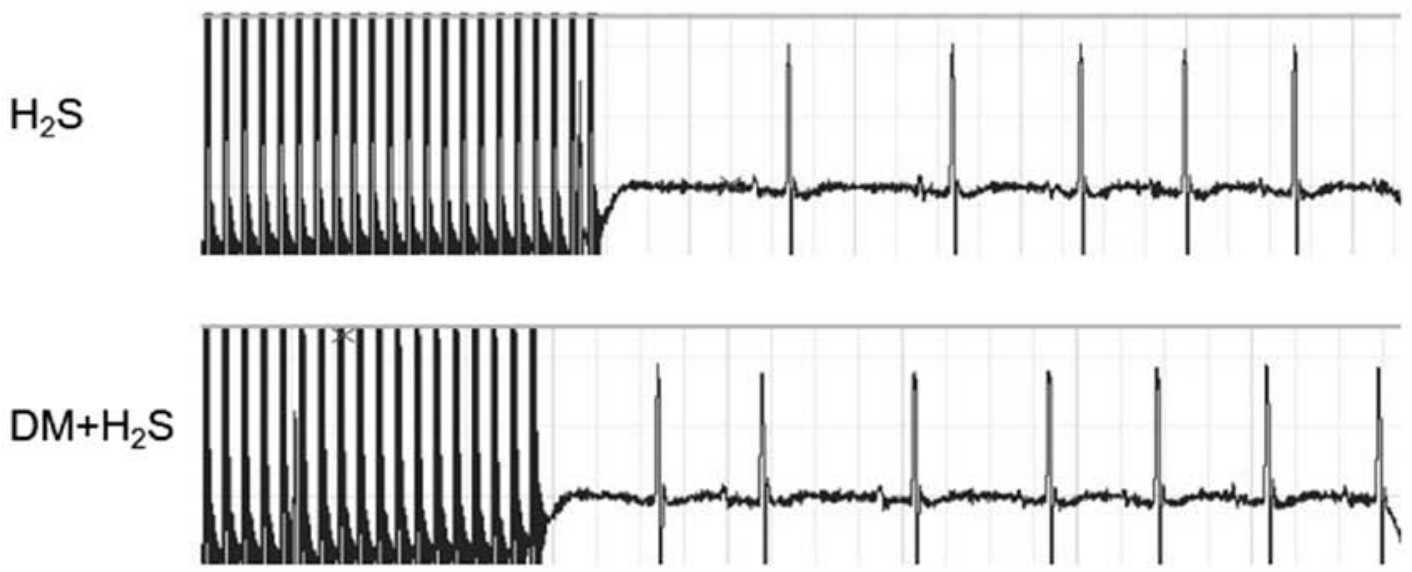

B

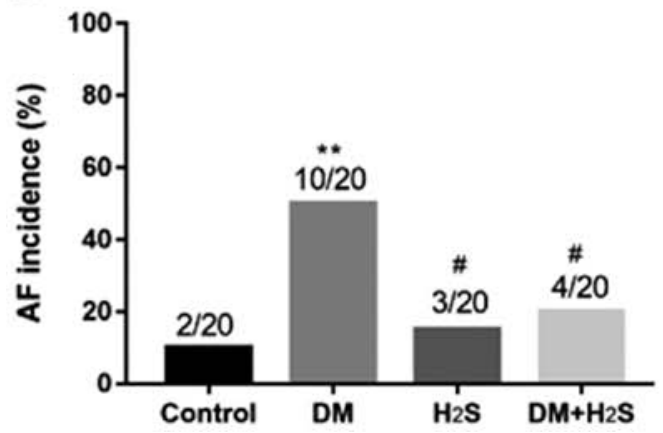

C

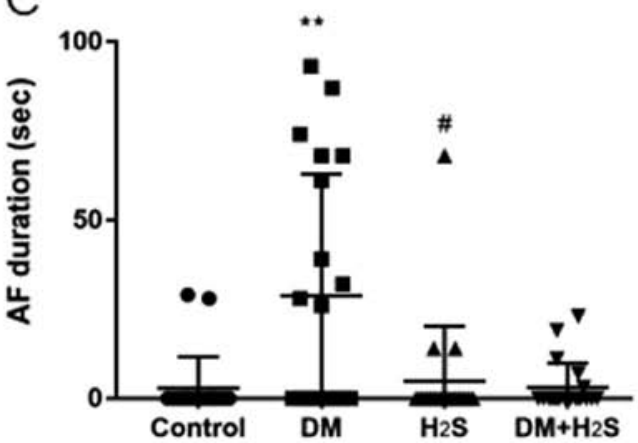

Figure 2. Intracardiac programmed electrical stimuli results. (A) Representative burst pacing electrocardiogram wave. (B) AF incidence in the four groups. (C) AF duration in the four groups. $\mathrm{n}=20$ for each group. Data are presented as mean $\pm \mathrm{SD}$. ${ }^{* *} \mathrm{P}<0.01$ vs. the control group; ${ }^{\#} \mathrm{P}<0.05$ vs. the $\mathrm{DM}$ group. DM, diabetes mellitus; AF, atrial fibrillation; $\mathrm{H}_{2} \mathrm{~S}$, hydrogen sulfide.

present study, SD rats were used as the research subject and a DM model was successfully established using STZ. It was demonstrated that DM rats had higher AF incidence and duration, which is associated with worse atrial fibrosis. It was also found that $\mathrm{H}_{2} \mathrm{~S}$ could reduce AF incidence and mitigate the atrial fibrosis, which was achieved via the PI3K/Akt/eNOS pathway. Although there was no statistical difference between the DM group and $\mathrm{DM}+\mathrm{H}_{2} \mathrm{~S}$ group in AF duration, which could be attributed to the sample size, we propose that $\mathrm{H}_{2} \mathrm{~S}$ could also shorten AF duration.

There are several ways to build DM animal models, including Zucker Diabetic Fatty rats, BioBreeding rats,
LEW.1AR1-iddm rats, Goto-Kakizaki rats, chemically induced diabetic models, and non-obese diabetic mouse and Akita mouse models (19). Hayami et al used STZ to damage pancreatic islet $\mathrm{B}$ cells and significantly increase blood glucose, and found that diabetic rats suffered severe inflammation and atrial fibrosis (5). The present study used the same models as Hayami, and found that diabetic rats had not only worse atrial fibrosis, but also higher AF incidence and duration. Notably, the application of STZ for the DM model causes islet B cell injury and a shortage of insulin, which is similar to type 1 diabetes without insulin resistance. Saito et al (4) used another diabetic rat model by administrating a high-fat 

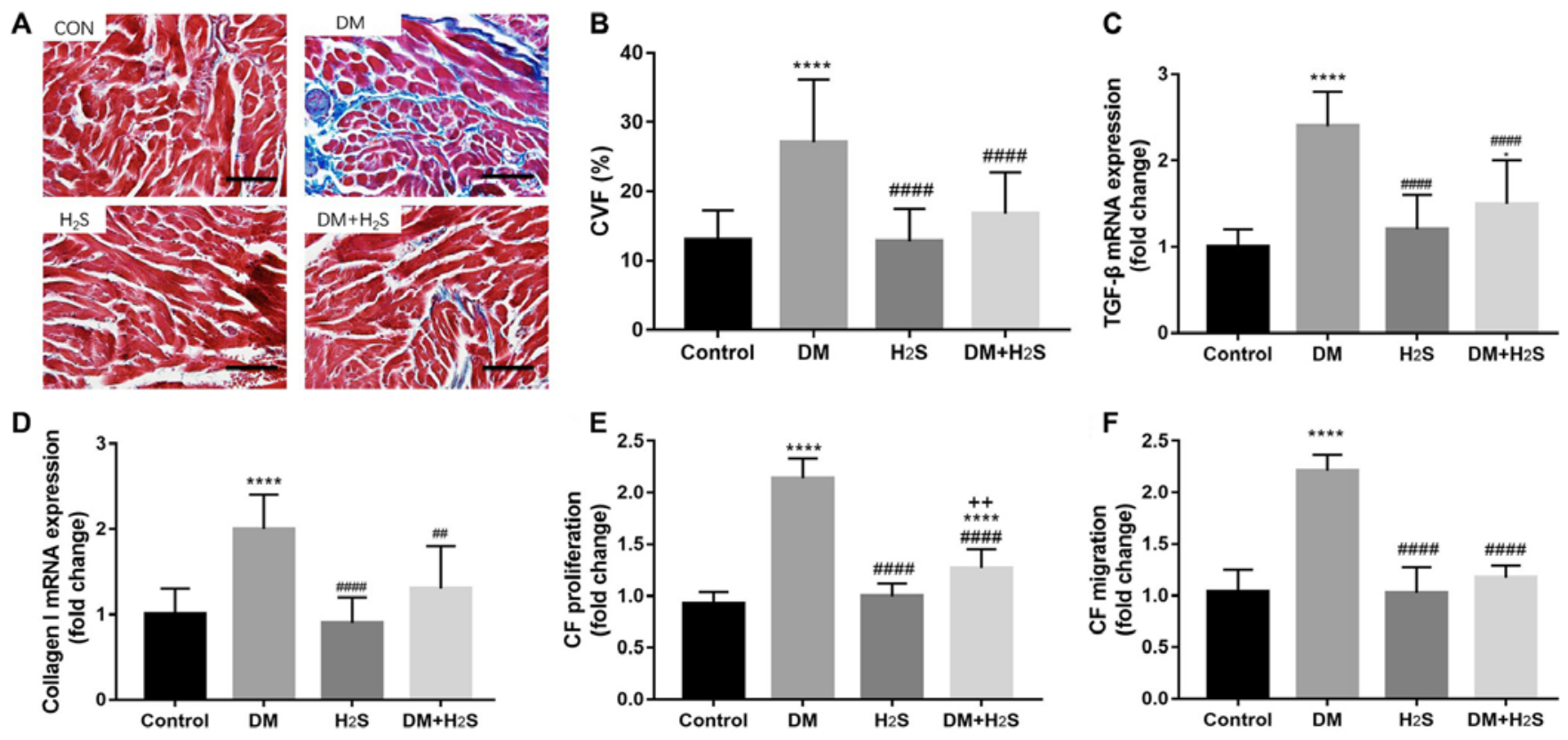

Figure 3. Atrial structural remodeling of fibrosis. (A) Representative Masson's trichrome staining sections of the four groups. Scale bar, $100 \mu \mathrm{m}$. (B) CVF $(\%)$ in the four groups. (C) TGF- $\beta$ mRNA expression in the four groups. (D) Collagen I mRNA expression in the four groups. (E) CF proliferation rate of the four groups. (F) CF migration rate of the four groups. $\mathrm{n}=20$ for each group. Data are presented as mean $\pm \mathrm{SD}$. ${ }^{*} \mathrm{P}<0.05$ and ${ }^{* * * * *} \mathrm{P}<0.0001$ vs. the control group; ${ }^{\# P} \mathrm{P}<0.01$ and ${ }^{\# \# \# \#} \mathrm{P}<0.0001$ vs. the $\mathrm{DM}$ group; ${ }^{++} \mathrm{P}<0.01$ vs. the $\mathrm{H}_{2} \mathrm{~S}$ group. Fold-change means the value compared with the control group. $\mathrm{CF}$, cardiac fibroblasts; CVF, collagen volume fraction; TGF- $\beta$, tumor growth factor $\beta$; DM, diabetes mellitus; $\mathrm{H}_{2} \mathrm{~S}$, hydrogen sulfide.
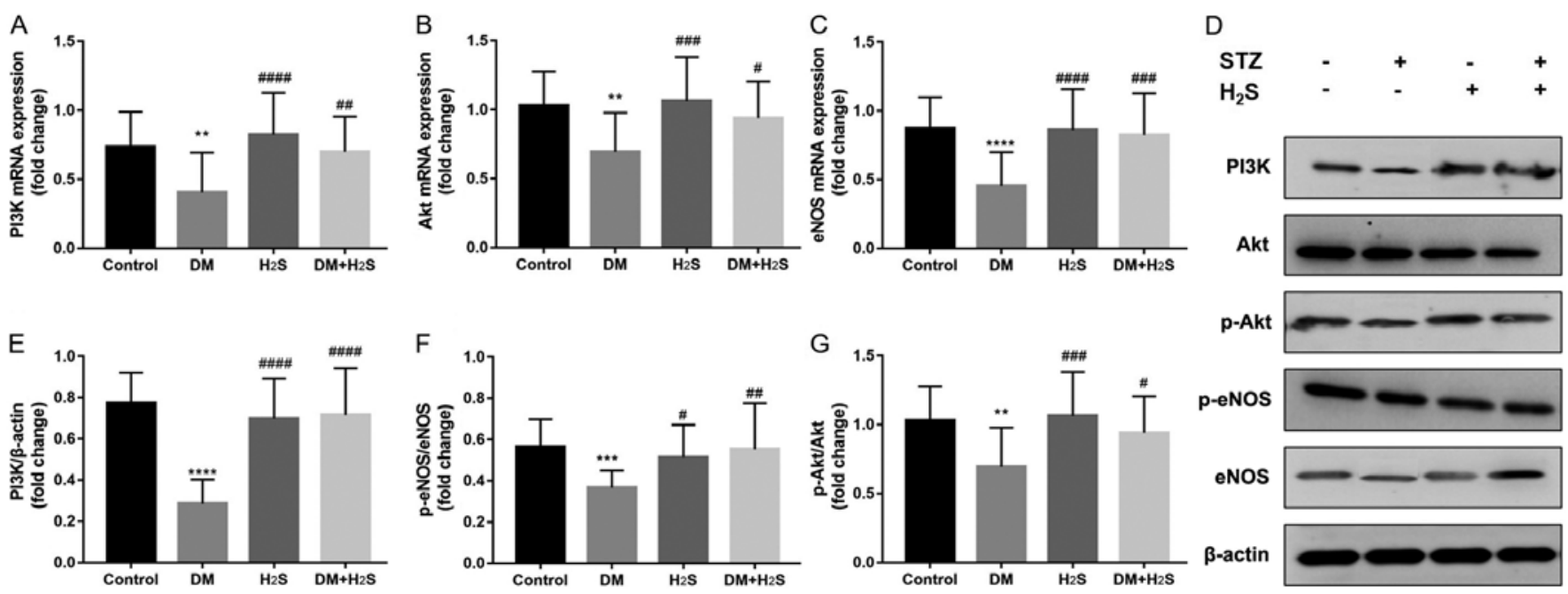

Figure 4. PI3k/Akt/eNOS pathway expression. (A) PI3K mRNA expression from atrium tissue normalized to $\beta$-actin. (B) Akt mRNA expression from atrium tissue normalized to $\beta$-actin. (C) eNOS mRNA expression from atrium tissue normalized to $\beta$-actin. (D) Representative western blotting bands of PI3K/Akt/eNOS pathway proteins. (E) PI3K protein expression level normalized to $\beta$-actin. (F) p-Akt/Akt protein level ratio. (G) p-eNOS/eNOS protein level ratio. $\mathrm{n}=20$ for each group. Data are presented as mean $\pm \mathrm{SD}$. ${ }^{* *} \mathrm{P}<0.01,{ }^{* * *} \mathrm{P}<0.001$ and ${ }^{* * * *} \mathrm{P}<0.0001$ vs. the control group; $\mathrm{P}<0.05,{ }^{\# \#} \mathrm{P}<0.01$, ${ }^{\# \# \#} \mathrm{P}<0.001$ and

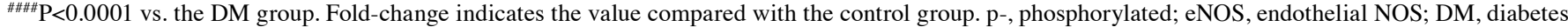
mellitus; $\mathrm{H}_{2} \mathrm{~S}$, hydrogen sulfide; STZ, streptozotocin.

diet and low-doses of STZ. In this way, the rats had the characteristics of insulin resistance and high blood glucose, which simulates type 2 diabetes.

Research on DM and AF has demonstrated that autonomic, electrical, electromechanical and structural remodeling, including oxidative stress, connexin remodeling and glycemic fluctuations, could be important reasons why patients with DM are at higher risk of AF (20). Among all the mechanisms, oxidative stress and related structural remodeling lay the foundation of abnormal electrical activity (6). Chang et al (21) led a large-scale cohort study and found that metformin use was associated with a decreased risk of AF in patients with type $2 \mathrm{DM}$ who were not using other anti-diabetic medication, most likely via attenuation of atrial cell tachycardia-induced myolysis and oxidative stress. Another study used the xanthine oxidase inhibitor allopurinol to prevent oxidative stress-mediated atrial remodeling in alloxan-induced DM rabbits (22). 
Activation of the PI3K/Akt/eNOS signaling pathway is associated with reduced reactive oxygen species production $(23,24)$. Chong et al $(25)$ found that resveratrol could decrease left atrial fibrosis and regulate variation in ion channels to reduce AF through the PI3K/Akt/eNOS signaling pathway. Hydrogen sulfide is closely associated with the PI3K/Akt pathway. Xiao et al (26) investigated the effects of hydrogen sulfide on myocardial fibrosis and found that the protective effect of $\mathrm{H}_{2} \mathrm{~S}$ against diabetes-induced myocardial fibrosis might be associated with the attenuation of autophagy via the upregulation of the PI3K/Akt1 signaling pathway. The present study found that $\mathrm{H}_{2} \mathrm{~S}$ could significantly upregulate the PI3K/Akt/eNOS signaling pathway and reduce atrial fibrosis induced by DM. However, Xiao et al (26) demonstrated decreased autophagy resulted from PI3K/Akt1 activation, which was not found in this study. Another study led by Liu et al (27) also confirmed that $\mathrm{H}_{2} \mathrm{~S}$ could ameliorate rat myocardial fibrosis induced by thyroxine, which may be associated with autophagy activated by upregulation of the PI3K/Akt signaling pathway and downregulation of miR-21, miR-34a and miR-214.

Several limitations of the present study must be noted. Firstly, despite the protective effect of $\mathrm{H}_{2} \mathrm{~S}$ on DM-induced AF and atrial fibrosis, further mechanisms, such as inflammation, apoptosis or autophagy, were not investigated in this study due to limited time and funds. Secondly, this study only used the STZ-induced DM model, which is similar to type 1 DM, so conclusions are limited. Finally, electrical remodeling and cardiac structural function could be altered by DM status, which was also not investigated. However, these limitations can be resolved in further studies.

In conclusion, DM can lead to the structural remodeling of atrial fibrosis, increasing AF incidence and persistence. Administration of $\mathrm{H}_{2} \mathrm{~S}$ does not affect glucose metabolism, but can significantly mitigate atrial fibrosis and reduce the incidence of AF, probably via the activation of the PI3K/Akt/eNOS pathway.

\section{Acknowledgements}

Not applicable.

\section{Funding}

This study was supported by the Outstanding Young Physician Program of Changzheng Hospital, Young Physician Startup Fund of Changzheng Hospital, and Project of Shanghai Science and Technology Commission (grant no. 17ZR1439100).

\section{Availability of data and materials}

The datasets used and or/analyzed during the current study are available from the corresponding author on reasonable request.

\section{Authors' contributions}

XX and JX designed the study. XX, QY and XL acquired and interpreted the data. WX, PW, and JS conducted the animal experiments. QY and JX prepared the manuscript and supervised the study protocol. All authors read and approved the manuscript and agree to be accountable for all aspects of the research in ensuring that the accuracy or integrity of any part of the work are appropriately investigated and resolved.

\section{Ethics approval and consent to participate}

The present study was approved by the Experimental Animal Administration Committee of the Second Military Medical University (Shanghai, China). Animal experiments were performed following the Guide for the Care and Use of Laboratory Animals (National Institutes of Health publication 85-23, revised 1996).

\section{Patient consent for publication}

Not applicable.

\section{Competing interests}

The authors declare that they have no competing interests.

\section{References}

1. Lip GY, Tse HF and Lane DA: Atrial fibrillation. Lancet 379: 648-661, 2012.

2. Bell DSH and Goncalves E: Atrial fibrillation and type 2 diabetes: Prevalence, etiology, pathophysiology and effect of anti-diabetic therapies. Diabetes Obes Metab 21: 210-217, 2019.

3. Proietti R, Russo V, Wu MA, Maggioni AP and Marfella R: Diabetes mellitus and atrial fibrillation: Evidence of a pathophysiological, clinical and epidemiological association beyond the thromboembolic risk. G Ital Cardiol (Rome) 18: 199-207, 2017 (In Italian)

4. Saito S, Teshima Y, Fukui A, Kondo H, Nishio S, Nakagawa M, Saikawa $\mathrm{T}$ and Takahashi N: Glucose fluctuations increase the incidence of atrial fibrillation in diabetic rats. Cardiovasc Res 104: 5-14, 2014.

5. Hayami N, Sekiguchi A, Iwasaki YK, Murakawa Y and Yamashita T: No additional effect of DPP-4 inhibitor on preventing atrial fibrosis in streptozotocin-induced diabetic rat as compared with sulfonylurea. Int Heart J 57: 336-340, 2016.

6. Karam BS, Chavez-Moreno A, Koh W, Akar JG and Akar FG: Oxidative stress and inflammation as central mediators of atrial fibrillation in obesity and diabetes. Cardiovasc Diabetol 16: 120, 2017.

7. Wu D, Wang J, Li H, Xue M, Ji A and Li Y: Role of hydrogen sulfide in ischemia-reperfusion injury. Oxid Med Cell Longev 2015: 186908, 2015.

8. Citi V, Piragine E, Testai L, Breschi MC, Calderone V and Martelli A: The role of hydrogen sulfide and H2S-donors in myocardial protection against ischemia/reperfusion injury. Curr Med Chem 25: 4380-4401, 2018.

9. Zhang FL, Chu SL, Wang WW and Chen LL: Downregulated PI3K-Akt-eNOS expression is related to increased atrial fibrillation inducibility in diabetic rats. Zhonghua Xin Xue Guan Bing Za Zhi 46: 376-381, 2018 (In Chinese).

10. Olas B: Hydrogen sulfide in signaling pathways. Clin Chim Acta 439: 212-218, 2015.

11. Toldo S, Das A, Mezzaroma E, Chau VQ, Marchetti C, Durrant D, Samidurai A, Van Tassell BW, Yin C, Ockaili RA, et al: Induction of microRNA-21 with exogenous hydrogen sulfide attenuates myocardial ischemic and inflammatory injury in mice. Circ Cardiovasc Genet 7: 311-320, 2014.

12. Sohrabipour S, Sharifi MR, Talebi A, Sharifi M and Soltani N: GABA dramatically improves glucose tolerance in streptozotocin-induced diabetic rats fed with high-fat diet. Eur J Pharmacol 826: 75-84, 2018.

13. Jin X, Jiang Y, Xue G, Yuan Y, Zhu H, Zhan L, Zhuang Y, Huang Q, Shi L, Zhao Y, et al: Increase of late sodium current contributes to enhanced susceptibility to atrial fibrillation in diabetic mice. Eur J Pharmacol 857: 172444, 2019. 
14. Luo T, Chen B and Wang X: 4-PBA prevents pressure overload-induced myocardial hypertrophy and interstitial fibrosis by attenuating endoplasmic reticulum stress. Chem Biol Interact 242: 99-106, 2015.

15. Livak KJ and Schmittgen TD: Analysis of relative gene expression data using real-time quantitative PCR and the 2(-Delta Delta C(T)) method. Methods 25: 402-408, 2001

16. Meng T, Cheng G, Wei Y, Ma S, Jiang Y, Wu J, Zhou X and Sun C: Exposure to a chronic high-fat diet promotes atrial structure and gap junction remodeling in rats. Int J Mol Med 40 217-225, 2017.

17. Shen H, Wang J, Min J, Xi W, Gao Y, Yin L, Yu Y, Liu K, Xiao J, Zhang YF and Wang ZN: Activation of TGF- $\beta 1 / \alpha-S M A / C o l ~ I$ profibrotic pathway in fibroblasts by galectin-3 contributes to atrial fibrosis in experimental models and patients. Cell Physiol Biochem 47: 851-863, 2018.

18. Ma J, Ma S, Yin C and $\mathrm{Wu} \mathrm{H}$ : Matrine reduces susceptibility to postinfarct atrial fibrillation in rats due to antifibrotic properties. J Cardiovasc Electrophysiol 29: 616-627, 2018.

19. Al-Awar A, Kupai K, Veszelka M, Szúcs G, Attieh Z, Murlasits Z, Török S, Pósa A and Varga C: Experimental diabetes mellitus in different animal models. J Diabetes Res 2016: 9051426, 2016.

20. Goudis CA, Korantzopoulos P, Ntalas IV, Kallergis EM, Liu T and Ketikoglou DG: Diabetes mellitus and atrial fibrillation: Pathophysiological mechanisms and potential upstream therapies. Int J Cardiol 184: 617-622, 2015.

21. Chang SH, Wu LS, Chiou MJ, Liu JR, Yu KH, Kuo CF, Wen MS, Chen WJ, Yeh YH and See LC: Association of metformin with lower atrial fibrillation risk among patients with type 2 diabetes mellitus: A population-based dynamic cohort and in vitro studies. Cardiovasc Diabetol 13: 123, 2014.
22. Yang Y, Zhao J, Qiu J, Li J, Liang X, Zhang Z, Zhang X, Fu H, Korantzopoulos P, Letsas KP, et al: Xanthine oxidase inhibitor allopurinol prevents oxidative stress-mediated atrial remodeling in alloxan-induced diabetes mellitus rabbits. J Am Heart Assoc 7: $\mathrm{e} 008807,2018$

23. Wang R, Peng L, Zhao J, Zhang L, Guo C, Zheng W and Chen H: Gardenamide a protects RGC-5 cells from H(2)O(2)-induced oxidative stress insults by activating PI3K/Akt/eNOS signaling pathway. Int J Mol Sci 16: 22350-22367, 2015.

24. Chu P, Han G, Ahsan A, Sun Z, Liu S, Zhang Z, Sun B, Song Y, Lin Y and Peng J, Tang Z: Phosphocreatine protects endothelial cells from Methylglyoxal induced oxidative stress and apoptosis via the regulation of PI3K/Akt/eNOS and NF- $\kappa \mathrm{B}$ pathway. Vascul Pharmacol 91: 26-35, 2017.

25. Chong E, Chang SL, Hsiao YW, Singhal R, Liu SH, Leha T, Lin WY, Hsu CP, Chen YC, Chen YJ, et al: Resveratrol, a red wine antioxidant, reduces atrial fibrillation susceptibility in the failing heart by PI3K/AKT/eNOS signaling pathway activation. Heart Rhythm 12: 1046-1056, 2015.

26. Xiao T, Luo J, Wu Z, Li F, Zeng $\mathrm{O}$ and Yang J: Effects of hydrogen sulfide on myocardial fibrosis and PI3K/AKT1-regulated autophagy in diabetic rats. Mol Med Rep 13: 1765-1773, 2016.

27. Liu M, Li Z, Liang B, Li L, Liu S, Tan W, Long J, Tang F, Chu C and Yang J: Hydrogen sulfide ameliorates rat myocardial fibrosis induced by thyroxine through PI3K/AKT signaling pathway. Endocr J 65: 769-781, 2018.

(i) () $९$ This work is licensed under a Creative Commons Attribution-NonCommercial-NoDerivatives 4.0 International (CC BY-NC-ND 4.0) License. 